\begin{tabular}{|c|ll|}
\hline $\begin{array}{c}\text { III Simpósio Paranaense de Modelagem, } \\
\text { Simulaçăoe Controle de Processos }\end{array}$ & Artigo: 37 \\
\cline { 3 - 3 } ISSN : $1984-7521$ & Páginas: & $274-279$ \\
\hline
\end{tabular}

\title{
DESENVOLVIMENTO DE UM MÓDULO EXPERIMENTAL DE CONTROLE DE TEMPERATURA - FEEDBACK E FEEDFORWARD
}

\author{
Roberto N. Koepsel*, Victor H. Barlatti, Marcos de Souza, Cid M. G. Andrade \\ 1 - Universidade Estadual de Maringá -UEM - PR, koepsel15@gmail.com \\ 2 - Universidade Estadual de Maringá - UEM - PR \\ 3 - Universidade Estadual de Maringá - UEM - PR \\ 4 - Universidade Estadual de Maringá - UEM - PR
}

\begin{abstract}
Resumo - Os sistemas de controle são essenciais para diminuir erros e aumentar a competitividade no mercado, nesse sentido a utilização de módulos experimentais tem possibilitado simular processos e formas de controle. Este artigo relata a construção de um módulo experimental de baixo custo baseado em softwares livres para ser utilizado em fins didáticos, simulando um processo de aquecimento. Com o experimento objetivou-se realizar a montagem de um tanque e conectá-lo a um sistema de medição de temperatura. $\mathrm{O}$ tipo de atuação e conexão com os sensores foi pensado para diminuir custos e facilitar a compatibilização entre a estrutura e os componentes eletrônicos. Conclui-se que o módulo permite estudar os melhores valores para as constantes de um controlador do tipo PID, além de permitir atuar de forma antecipatória, pois analisa os sensores da entrada e encontra todas as variáveis presentes.
\end{abstract}

Palavras-chave: Controle. Modulo experimental de controle. Controle PID. Feedforward. Feedback. Aquecimento.

\section{Introdução}

Os sistemas de controle são cada vez mais necessários para diminuir erros e aumentar a competitividade no mercado, cada vez mais concorrido [1]. Fato esse tem estimulado, a utilização de módulos experimentais que permitem simular um processo e suas mais variadas formas de controle. Trata-se da aplicação prática dos conceitos teóricos. As informações obtidas nos testes permitem cálculos mais precisos que objetivam máxima economia, por exemplo.

Tendo em mente a existência de sistemas de controle de malha aberta e fechada, sabendo que em um sistema de malha aberta a saída não é medida e que em um sistema de malha fechada o sistema alimenta o controlador com as informações da saída, é fundamental conhecer suas vantagens e suas limitações. [3]

Para um sistema de malha aberta a calibração do controlador é fundamental, em consequência de não ser possível corrigir possíveis distúrbios não previstos no processo. Contudo, tem a vantagem de não alterar a estabilidade do sistema e também de possuir uma construção mais simples e de baixo custo. Já o sistema de malha fechada permite uma aplicação em sistemas no qual não é possível prever todas as variações que sejam capazes de ocorrer no processo, uma vez que a saída do sistema realimenta o controlador e gera novas ações de controle. [2]

Este artigo relata a construção de um modulo experimental de baixo custo baseado em softwares livres para ser utilizado em fins didáticos, simulando um processo de aquecimento. Buscou-se reproduzir no módulo desenvolvido uma experiência mais 
próxima do que ocorre na indústria. Para tanto, objetivou-se realizar a montagem de um tanque e conectá-lo a um sistema de medição de temperatura, assim como desenvolver um controlador que possa através de sua interface monitorar o sistema de forma antecipatória ou utilizando uma realimentação.

\section{Materiais e métodos}

O módulo experimental simula um processo de aquecimento que permite inúmeros tipos de controles, tanto em malha aberta quanto para processos em malha fechada. Como pode ser visto na figura 1, conta-se com sensores de temperatura na entrada e na saída do sistema; um módulo responsável por realizar o controle; uma resistência elétrica como variável manipulada e, para testar sua eficiência, outra resistência elétrica foi instalada na entrada do processo para simular possíveis perturbações.

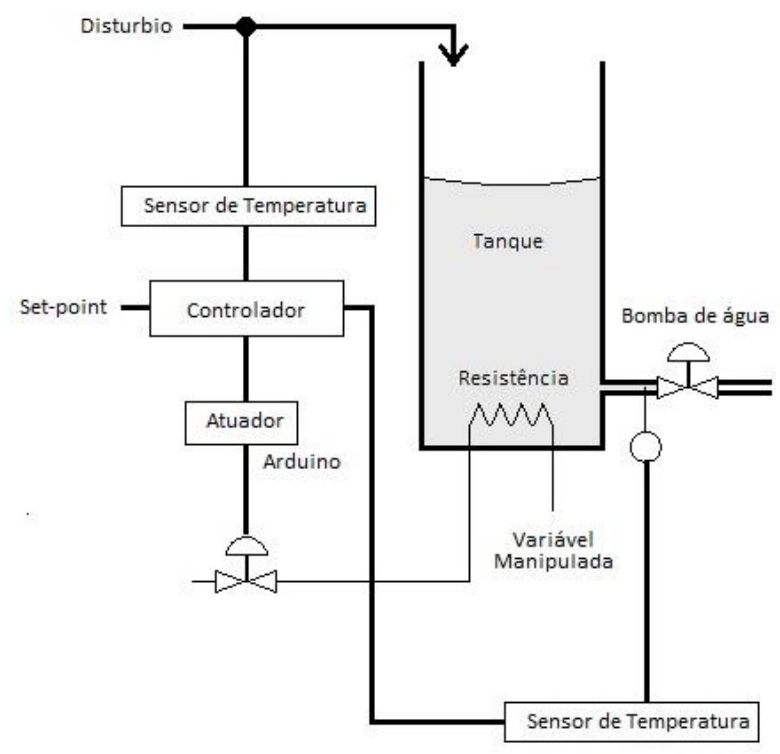

Figura 1- Diagrama completo do sistema.

A construção do equipamento demandou a escolha dos materiais pertinentes a cada parte do experimento, isso se refere à estrutura e aos componentes eletrônicos. $\mathrm{O}$ tipo de atuação e conexão com os sensores foi pensado para diminuir custos e facilitar a compatibilização entre eles.

Para receber os dados de temperatura foi utilizado o sensor LM35, pois ele apresenta uma precisão de $0,25^{\circ} \mathrm{C}$ a $0,75^{\circ} \mathrm{C}$ e pode trabalhar em temperaturas de até 150 ${ }^{\circ} \mathrm{C}$, além de ser de fácil aquisição e compatível com as entradas do Arduino UNO, responsável por converter seus sinais. [4]

O tanque foi confeccionado em Acrílico do tipo Cast, pois possui grande durabilidade e transparência, características essenciais para o experimento. $\mathrm{O}$ material escolhido é mais leve e mais econômico que um tanque equivalente em vidro.

O programa utilizado para programar o controlador foi o software Scilab, ele através de uma ligação serial com o Arduino recebe, processa e envia o sinal de controle 
para o atuador, nele, por meio de uma interface o operador pode definir os parâmetros que deverão ser seguidos pelo sistema, como o tipo de controle e suas constantes.

A ligação entre os sensores foi feita utilizando o Arduino Uno, ele é o responsável por converter o sinal analógico recebido dos sensores de temperatura e enviar o sinal digital, através de uma comunicação serial, para o controlador presente no Scilab.

Para atuar no sistema foi necessário conectar o Arduino a um circuito, como visualizado na figura 2. Através de um sinal PWM (Pulse-Width Modulation ou modulação por largura de pulso) enviado pelo Arduino é possível transmitir a potência definida pelo controlador à resistência elétrica presente no tanque.

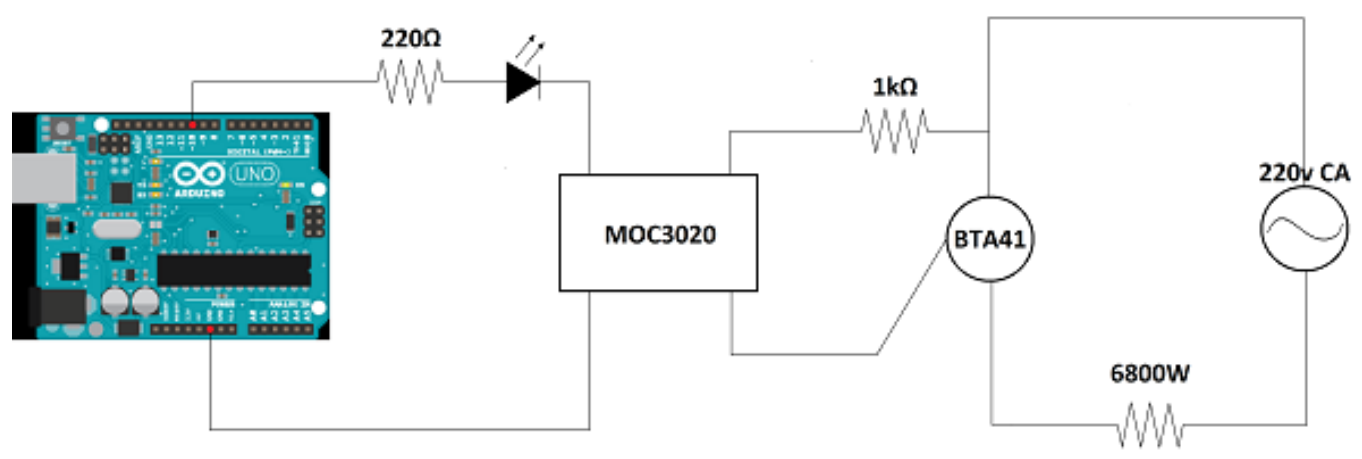

Figura 2 - Circuito de controle PWM

Com o sistema pronto, ilustrado na figura (3), foi possível fazer a modelagem do tanque e obter a função de transferência, que futuramente poderá ser utilizada para a sintonia do controlador.

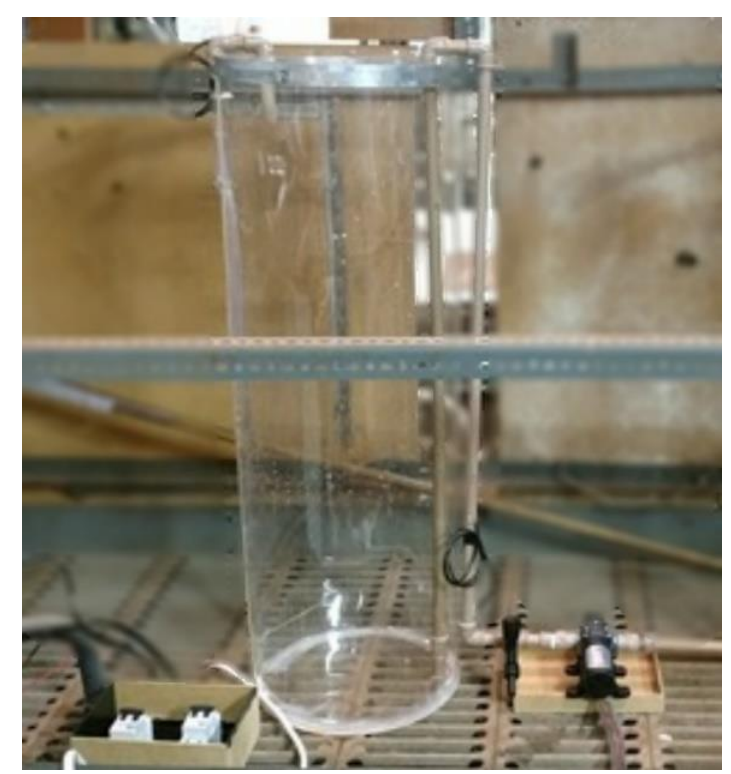

Figura 3 - Módulo montado

\section{Resultados e discussão}

Os resultados obtidos podem ser vistos na interface criada para analisar os dados de entrada e saída do sistema, assim como a resposta do sistema e o valor definido para o setpoint no início do processo, os quais podem ser visualizados na figura 4 . 


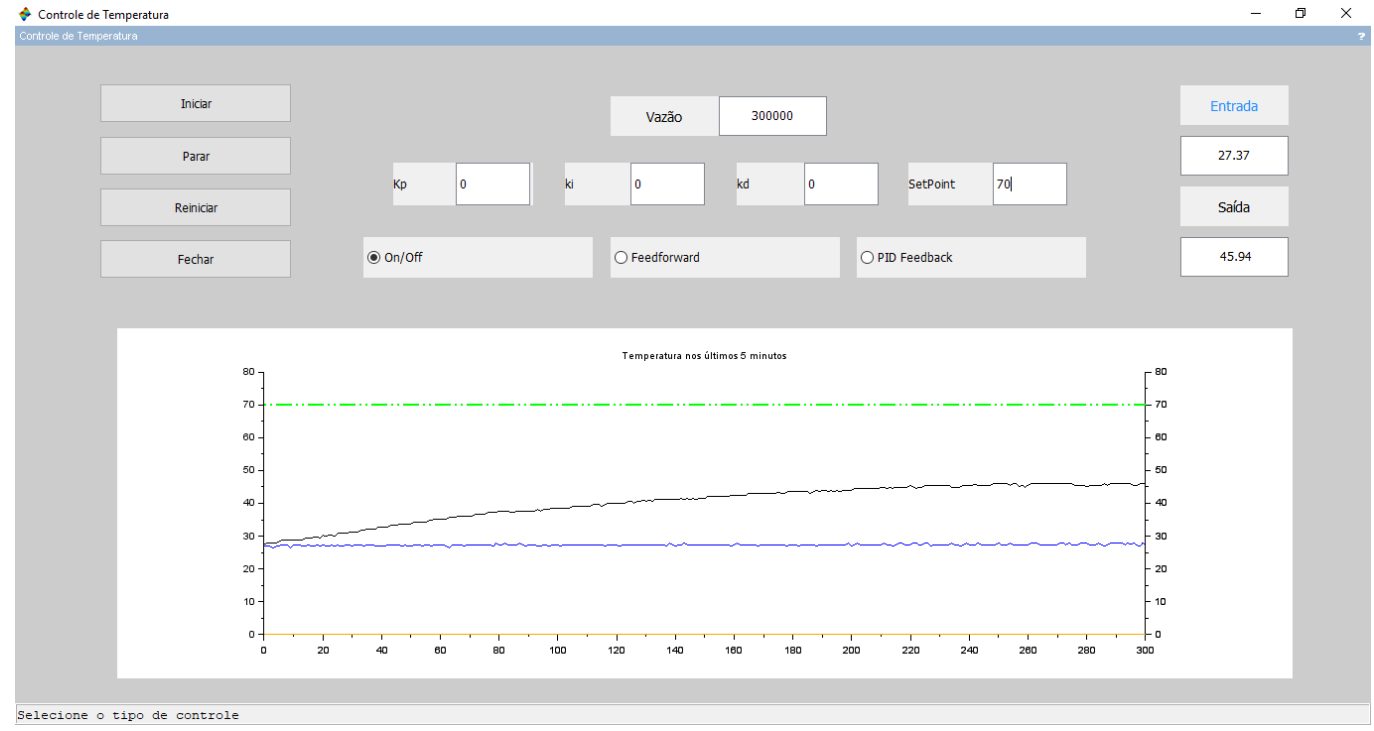

Figura 4 - Interface para receber e visualizar os dados do sistema

Os campos disponibilizados para o usuário foram os valores das constantes PID assim como o tipo de controle e a vazão do sistema durante a realização do experimento. No próprio programa também é possível enviar os dados obtidos para outro software para serem feitas as análises necessárias.

Através do programa desenvolvido foi possível identificar experimentalmente o ganho do sistema. Para obter seu valor foi criado um gráfico no qual foi obtido o ganho de temperatura para cada potência definida, no gráfico (1) realizou incrementos de $10 \%$ até atingir a potência máxima do sistema. A escala utilizada foi de 0 a 255 que é a máxima divisão permitida pela Arduino, sendo 255 equivalente a 6800Watts, potência máxima da resistência elétrica do sistema.

\section{Gráfico 1 - Ganho do sistema}

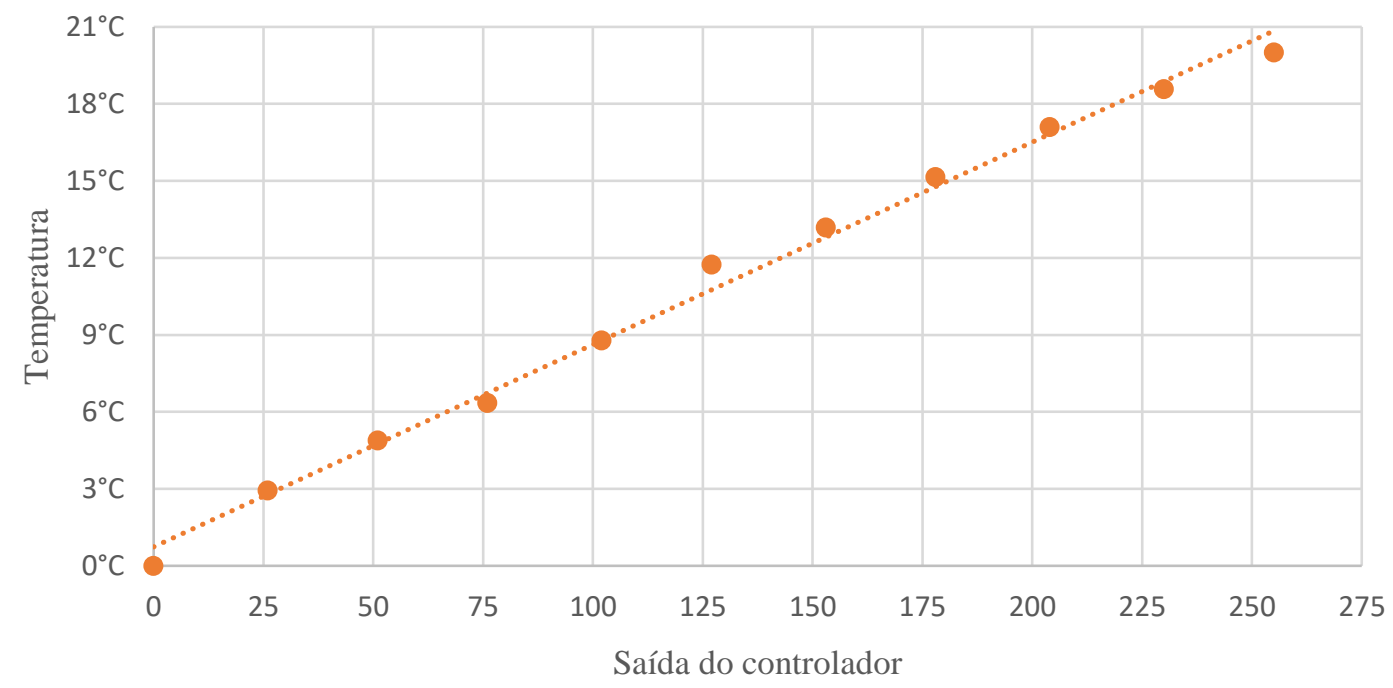

Através da resposta do sistema a um degrau na resistência é possível também identificar a constante de tempo do sistema, que equivale a $63,2 \%$ da temperatura a ser atingida. Como pode ser visto no gráfico 2. 
Gráfico 2 - Resposta ao degrau de temperatura

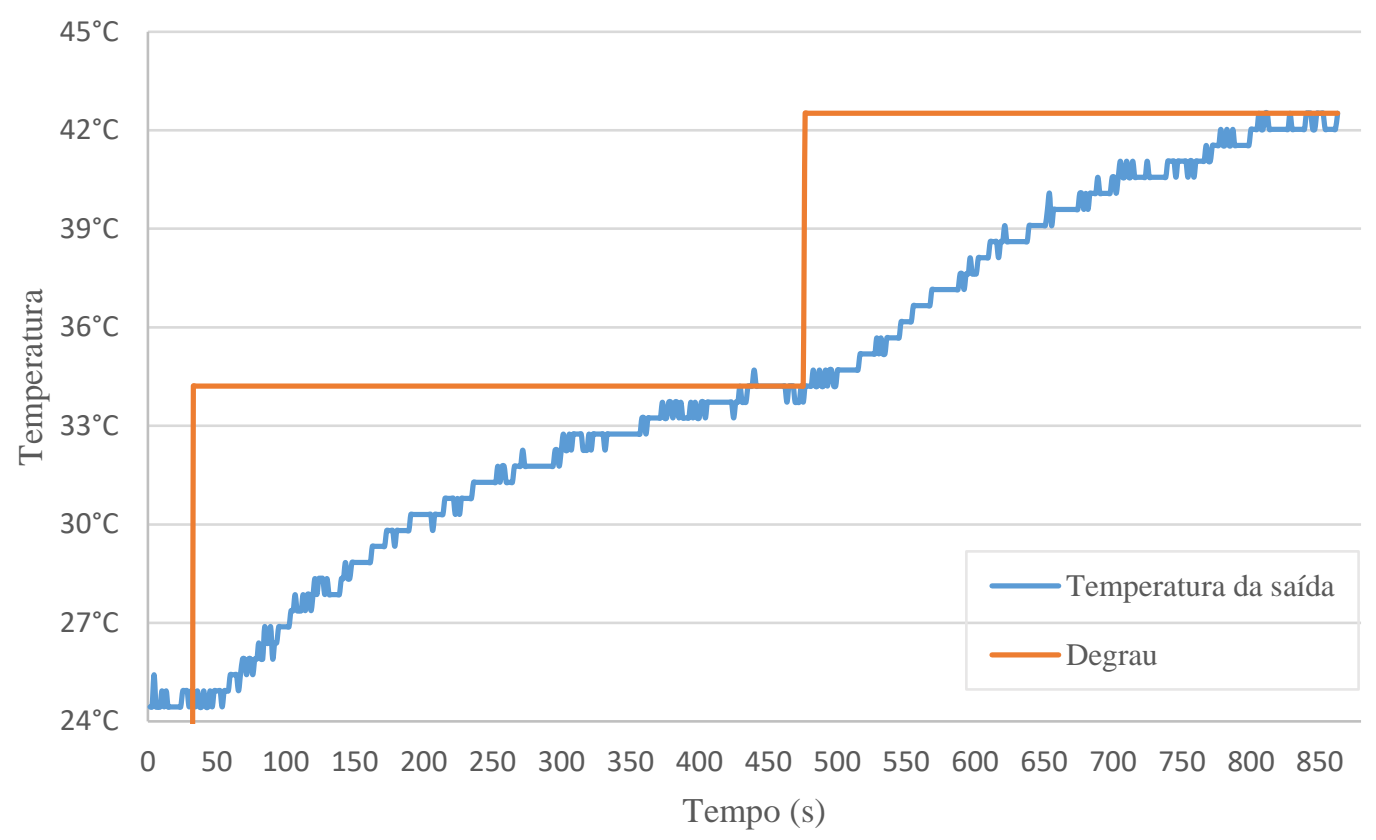

A partir da resposta recebida é obtida a função de transferência do sistema. A equação é dada pelo ganho do sistema e pelo tempo de resposta do sistema. Através dos gráficos 1 e 2 resulta em:

$$
G(s)=\frac{0.003}{180 s+1}
$$

\section{Conclusão}

O trabalho resultou em uma planta que permite simular inúmeras perturbações e controlar um processo de aquecimento. O módulo permite estudar os melhores valores para as constantes de um controlador do tipo PID, além de permitir atuar de forma antecipatória, pois analisa os sensores da entrada e encontra todas as variáveis presentes.

No sistema criado é possível futuramente adicionar outras variáveis de um processo, como exemplo, o nível do líquido, uma vez que o controlador nem o atuador foram saturados, o qual permitiria inserir novos instrumentos de medição e controle ao processo.

\section{Referências}

[1] SENAI. Instrumentação: Fundamentos de Controle de processo. Departamento Regional do Espírito Santo. CPM - Programa de Certificação do Pessoal de Manutenção. Espírito Santo, 1999.

[2] OGATA, Katsuhiko. Engenharia de controle moderno. 4. ed. [S.1.]: Pearson, 2003.

[3] OLIVEIRA, Adalberto Luiz de Lima et al. (Org.). Fundamentos de controle de processo. ES: SENAI, 1999. 
[4] TEXAS INSTRUMENTS INCORPORATED. (Dallas, Texas). LM35 Precision Centigrade Temperature Sensors. Disponível em: <http://www.ti.com/product/LM35/datasheet〉. Acesso em: 10 out. 2017. 